\title{
Effect of Preamplifier on Timing Recovery in Magnetic Recording Channels
}

\author{
Raman Venkataramani, M. Fatih Erden, and Erozan Kurtas \\ Seagate Research \\ 1251 Waterfront Place, Pittsburgh, PA 15222
}

\begin{abstract}
We study the effect of the preamplifier cutoff frequency on the timing recovery performance of perpendicular magnetic recording systems with independent and identically distributed (IID) random input bits as well as run lengthlimited (RLL) encoded inputs. We observe that a larger cutoff frequency has a detrimental effect on the system performance. When using an RLL encoder with an IID input we observe a slight loss in performance. However, the true benefit of RLL codes is to prevent catastrophic failures for the worst-case inputs.
\end{abstract}

\section{Introduction}

A magnetic recording system may be viewed as a communication channel if we identify the write-head as the transmitter and the read-head as the receiver. There is a fundamental requirement that receiver and transmitter operate according to a common clock. While, the receiver generally knows the nominal values of the transmission frequency, the receiver clock is never truly synchronized with the transmitter clock. Thus, the receiver needs to recover the clock associated with the received signal from the signal itself. This problem of recovering the correct timing information from the received signal is called timing recovery or clock synchronization. In magnetic recording, the desire to push recording densities higher has caused today's recording devices to operate at low signal-to-noise ratio (SNR) and more sophisticated coding algorithms, while timing recovery itself becomes more challenging. Consequently, timing recovery is an important aspect in magnetic recording channels.

The purpose of this paper is to study the performance of the timing recovery in the system for different preamplifiers. The preamplifier is a component whose function is to amplify the weak readback signals originating from the read head prior to further signal processing. The preamplifier is typically a high pass filter, i.e., it does not pass the DC component. Consequently, some of the signal spectrum near the origin is filtered out. Although the preamplifier is an essential component of the read channel architecture, there are few studies on its effect on the system performance. We study the performance for both uncoded inputs as well as run length limited (RLL) inputs. Classically, RLL codes are used for introducing transitions to help timing recovery.

\section{Channel and Signal Model}

Figure 1 depicts a magnetic recording system. The input to the system $\left\{a_{n}\right\}$ denotes the actual bits that we write to the recording medium and the block labeled "channel" includes the physical components of the system such as the read and write head and the recording medium. We assume that this block also includes a preamplifier.

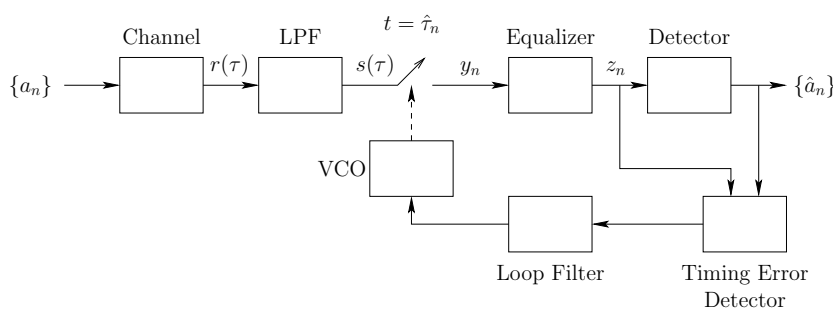

Figure 1. Conventional timing recovery.

We model the ideal readback waveform as follows

$$
r(t)=\sum_{m} b_{m} h_{T}(t-m T)+z(t)
$$

where $b_{m}=\left(a_{m}-a_{m-1}\right) / 2$ is the transition sequence corresponding to the written bit sequence $\left\{a_{m}= \pm 1\right\}$ which represents the magnetization of the medium, $T$ is the bitwidth, $h_{T}(t)$ is the transition response of the system defined as the response to a transition of the magnetization from $-1 \rightarrow+1$, and $z(t)$ is the additive Gaussian electronic noise with power spectral density of height $N_{0} / 2$. The above model ignores the effects of transition noise in the channel. 
For perpendicular recording, the transition response is

$$
h_{T}(t)=\operatorname{erf}\left(\frac{2 t \sqrt{\ln 2}}{W}\right)
$$

where

$$
\operatorname{erf}(x)=\frac{2}{\sqrt{\pi}} \int_{0}^{x} e^{-t^{2}} d t
$$

is the error function and $W$ is the pulse width defined by the limits $t= \pm W / 2$ where the derivative of the transition response is one half of its peak value. For longitudinal recording

$$
h_{T}(t)=\frac{1}{1+(2 t / W)^{2}}
$$

where $W$ is the pulse width defined by the limits $t= \pm W / 2$ where the transition response is one half of its peak value. The quantity $W$ is the " $50 \%$ pulse width" and is often written as PW50.

In the presence of transition jitter noise in the channel, the readback signal is given by

$$
r(t)=\sum_{m} b_{m} h_{T}\left(t-m T-\Delta t_{m}\right)+z(t)
$$

where $\Delta t_{m}$ is the transition jitter noise if there is a transition at time $m T$. The transition noise is modeled as an independent and identically distributed (IID) truncated Gaussian random variable with variance $\operatorname{var}\left(\Delta t_{m}\right)=\sigma_{j}^{2}$.

We adopt the following definition of signal-to-noise ratio (SNR) of the channel [1]:

$$
\mathrm{SNR}=\frac{E_{i}}{N_{0}+M_{0}}
$$

where $E_{i}$ is the energy in the derivative of the transition response $h_{T}(t)$ scaled by $W^{2}$ to preserve the units of energy

$$
E_{i}=W^{2} \int_{\mathbb{R}}\left|h_{T}^{\prime}(t)\right|^{2} d t
$$

and $M_{0} / 2$ is the average transition noise energy per bit which is obtained by using a first order Taylor series approximation to separate the effect of the transition noise from the clean signal.

$$
M_{0}=\frac{E_{i} \sigma_{j}^{2}}{W^{2}} .
$$

We model the preamplifier as a simple first-order high pass filter with cutoff frequency $\omega$ :

$$
H_{\text {pre }}(s)=\frac{s}{s+\omega} .
$$

Assuming that the channel response is essentially bandlimited to the frequencies, $[-1 / 2 T, 1 / 2 T]$, we can apply front-end analog low-pass filter with cut-off frequencies $\pm 1 / 2 T$ to obtain the filtered readback waveform. Furthermore, the sampling theorem states that the samples of this waveform at $t=n T, n \in \mathbb{Z}$ provide sufficient statistics. However, these are not the correct sampling times due to timing error in the system. The correct sampling locations are determined by the timing recovery blocks from the signal itself. The sampled sequence is then passed through an equalizer whose function is to reduce the inter-symbol interference (ISI) in the system to a few symbols. Finally, the detector computes estimates $\hat{a}_{n}$ of the input bits.

\subsection{Timing Recovery}

Traditional timing recovery architectures consist of a timing error detector whose function is to process the received samples to produce a quantity that is a measure of the timing phase error. This is further passed through a loop filter to produce adjustments to the timing phase, which in turn drives the sampler through a voltage controlled oscillator (VCO). The system is decision-directed, i.e., the detected bits are used by the timing recovery algorithm with the assumption that they are error free.

The system in Figure 1 uses a conventional timing recovery system. A widely used timing error detector in many practical systems is the Mueller and Müller (MM) detector [2] which computes the quantity

$$
e_{n}=\frac{1}{2}\left(z_{n} \hat{a}_{n-1}-z_{n-1} \hat{a}_{n}\right)
$$

where $z_{n}$ is the equalized sequence, and $\hat{a}_{n}$ is the detected sequence. The quantity $e_{n}$ is a measure of the local timing phase error. This is filtered using a loop filter whose response is

$$
H(z)=\alpha+\frac{\beta}{1-z^{-1}}
$$

for appropriate loop filter coefficients. This in turn controls a voltage controlled oscillator that triggers the sampler.

A thorough discussion of various timing recovery algorithms can be found in [3]. In this paper, we focus on MM timing recovery alone.

\subsection{Run Length Limited Codes}

In magnetic recording systems bits written on the medium are represented by the two magnetization levels of \pm 1 . All the bits occurring between two consecutive transitions in a bit sequence is called a run. The number of bits in a run is called the run length. A run length limited code is a code that constrains the run lengths to be smaller than some number so that the transitions in the bit sequences have desired characteristics.

RLL sequences are characterized by two parameters which specify the minimum and maximum run lengths contained in the sequence. A $(d, k)$ limited binary sequence 
is an RLL sequence whose minimum run length is $d+1$ and maximum run length is $k+1$. A collection of such sequences yields a $(d, k)$ RLL code.

Run length limited codes have been widely used in optical and disc recording systems. Classically, the use of RLL codes is to introduce frequent transitions (by means of the $k$-constraint) to help with timing recovery, while the $d$-constraint is used to reduce the ISI. It should be noted that a large $d$ constraint reduces the code rate significantly. A thorough discussion of RLL codes can be found in [4].

\section{Simulation Results}

In this section, we evaluate the performance of the timing estimation algorithm for uncoded and RLL coded magnetic recording channel by computer simulation. We consider perpendicular recording at a normalized recording density $W / T=2$. In our simulations, we process sectors consisting of 4096 information bits and an additional 100 bits for the preamble. We use an equalizer of length 11 designed for a monic generalized partial response (GPR) target [5] of length 3, and a Viterbi detector with detection depth of 4 bits.

In our simulations, we choose $T=1$ without loss of generality. The actual frequency offset encountered in a real system is the range of $0.2 \%$ to $0.4 \%$ and the initial phase offset is about 0.5 of a clock pulse. In all the simulations we artificially add an initial phase offset of $\tau_{0}=0.5 T$ and a clock frequency offset of $0.5 \%$. The jitter noise in the system is set at $90 \%$ of the total noise, i.e., $M_{0}=0.9\left(M_{0}+\right.$ $\left.N_{0}\right)$.

\subsection{Uncoded input}

We first consider the effect of the preamplifier filter for a system with uncoded (IID) input bits. We consider two preamplifiers whose normalized cutoff frequencies are $1 / 1000$ and $1 / 200$, i.e.,

$$
\omega=0.001 / T \text { and } \omega=0.005 / T \text {. }
$$

Figure 2 shows the following performance metrics: (a) bit error rate (BER), (b) cycle slip rate (CSR), and (c) timing error variance (normalized by the bit width) for each of the two preamplifier cutoff frequencies and various SNRs. A cycle slip is said to occur when the errors in the timing recovery phase locked loop cause the sampling phase to lose synchronization [6]. We declare a cycle slip if estimated sampling time is different from the ideal sampling time by more than half a bit for 100 consecutive samples. The BER includes the errors in those sectors with cycle slips. These results were obtained by processing 1000 sectors per operating point.
From the plots in Figure 2, we observe that, there is a performance loss due to a larger preamplifier cutoff frequency. This is due to the fact that for perpendicular recording there is significant signal energy near DC, which is filtered out by the preamplifier.

\subsection{RLL encoded input}

Transitions in the input bit sequences are important because this is where the timing information resides. We use RLL codes to encode the user bits to produce frequent transitions. We now evaluate the system performance in the presence of an RLL encoder for both the preamplifier cutoff frequencies. We choose a simple $(d, k)$ RLL code with $d=0$ and $k=3$. This code guarantees that the intertransition spacing is no more than $k+1=4$, but there is no constraint on the minimum spacing between transitions. This code has a rate of $8 / 9$.

Figure 3 shows the BER, CSR, and timing error variances for various SNR values for each of the preamplifier filters. The BER values correspond to the channel without the RLL code itself, i.e., they computed before the RLL decoding. As in the previous case, there is a loss in performance for the system with the larger preamplifier cutoff frequency. However, the performance gap is slightly smaller for the RLL coded case than for the uncoded case. This is presumably because the RLL encoded sequences have less energy at low frequencies than IID sequences and the preamplifier has a less pronounced effect on these signals.

Figure 4 shows the BER and CSR performance for above the uncoded and RLL encoded inputs for a preamplifier with cutoff frequency $0.005 / T$. It is apparent that adding the RLL code worsens the performance of the system. This is not unexpected and can be explained as follows. RLL codes are designed to improve the worst-case performance of the timing recovery by eliminating the "bad sequences" which have few or no transitions at all. Since our simulation inputs are IID, we don not see an improved performance with the RLL code. In fact we have a slightly worse performance with an RLL code. This is because the channel response for perpendicular recording is a low pass function, but the spectrum of the RLL code output has diminished low frequency components because of the frequent transitions in the output. The result is that the readback signal power is smaller for the RLL coded case, and this is reflected in the BER and CSR performance.

\section{Conclusions and Future Work}

We studied the effect of the preamplifier cut of frequency on the timing recovery performance of a perpendicular magnetic recording system. As expected, a larger cutoff frequency translated to worse performance. We also studied 
the effect of a simple RLL code on the timing recovery performance of the system. RLL codes are used primarily to make the timing recovery robust to worst-case inputs such as those with few transitions (or long run lengths).

Preliminary results with a simple RLL code show that when the user input is random and IID, the system performance is slightly worse than the corresponding uncoded case. Nevertheless, we must use RLL codes in recording to eliminate the worst case sequences. We also observe that RLL codes add some robustness to the effect of the preamplifier cutoff frequency.

As future work, we shall look at the performance other timing recovery algorithms such as interpolated timing recovery [7] and per-survivor processing [8].

\section{References}

[1] B. Vasic and E. Kurtas, Eds., Coding and Signal Processing for Magentic Recording Channels, CRC Press, 2005.

[2] K. Mueller and M. Müller, "Timing recovery in digital synchronous data receivers," IEEE Trans. on Comm., vol. COM-24, no. 5, pp. 516-531, May 1976.

[3] H. Meys, M. Moeneclaey, and S. A. Fechtel, Digital Communication Receivers, New York, USA, Wiley and Sons, 1998.

[4] K. A. Schouhamer Immink, Codes for Mass Data Storage, Shannon Foundation Publishers, 1999.

[5] J. Moon and W. Zeng, "Equalization for maximum likelihood detectors," IEEE Trans. Magnetics, vol. 31, no. 2, pp. 1083-1088, March 1995.

[6] J. W. M. Bergmans, Digital Baseband transmission and recording, Kluwer Academic Publishers, 1996.

[7] P. Kovintavewat, M. F. Erden, E. Kurtas, and J. R. Barry "Investigation of interpolated timing recovery for perpendicular recording channels," Seagate Internal Report.

[8] P. Kovintavewat, J. R. Barry, M. F. Erden, J. Park, E. Kurtas "Per-survivor timing recovery for uncoded partial response channels," Proc. Int. Conf. Commun. (ICC2004), Paris, France, June 20-24, 2004.

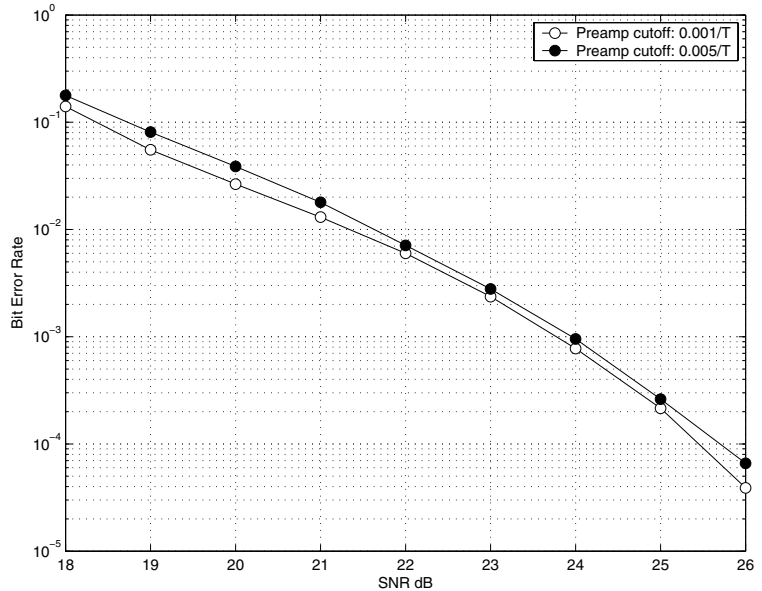

(a) Bit error rates.

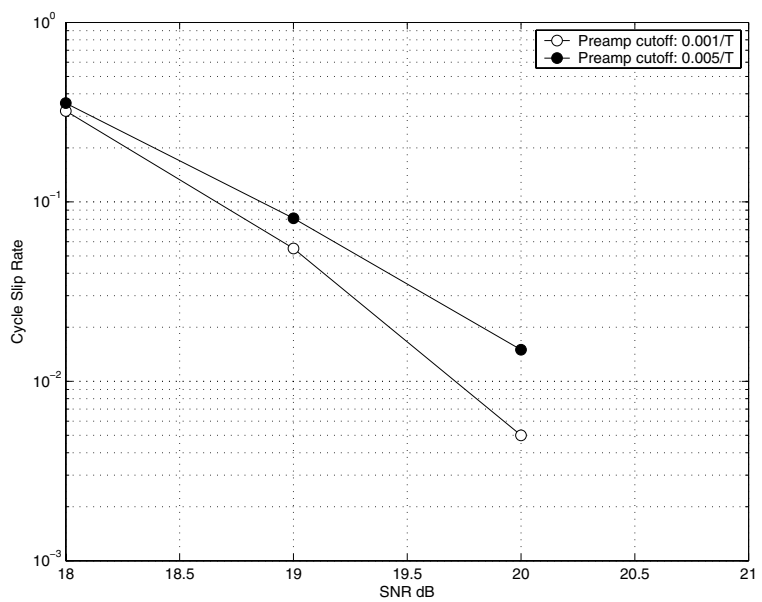

(b) Cycle slip rates.

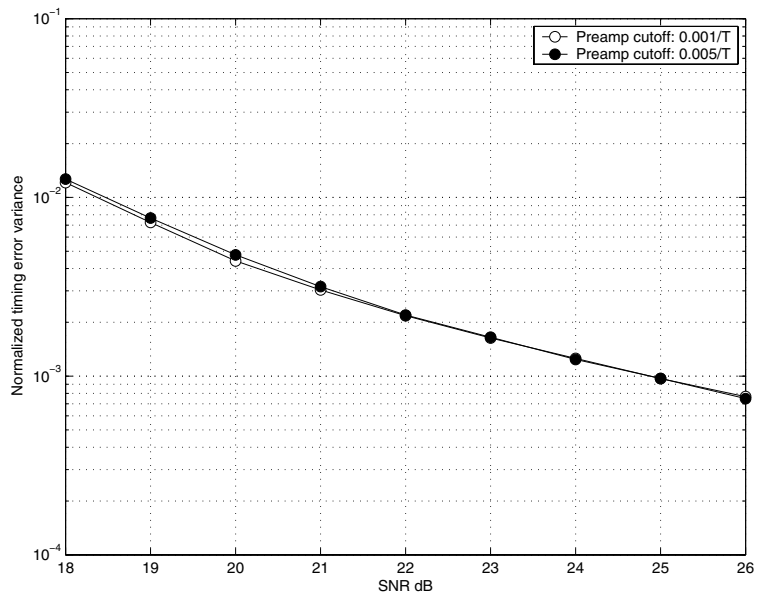

(c) Normalized timing error variances.

Figure 2. Comparison of timing recovery of two systems with different preamplifier cutoff frequencies and uncoded IID inputs. 


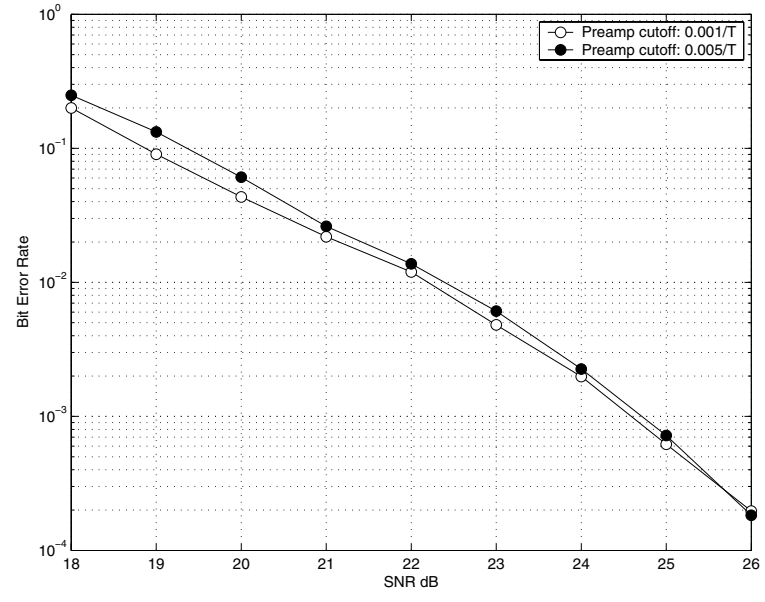

(a) Bit error rates.

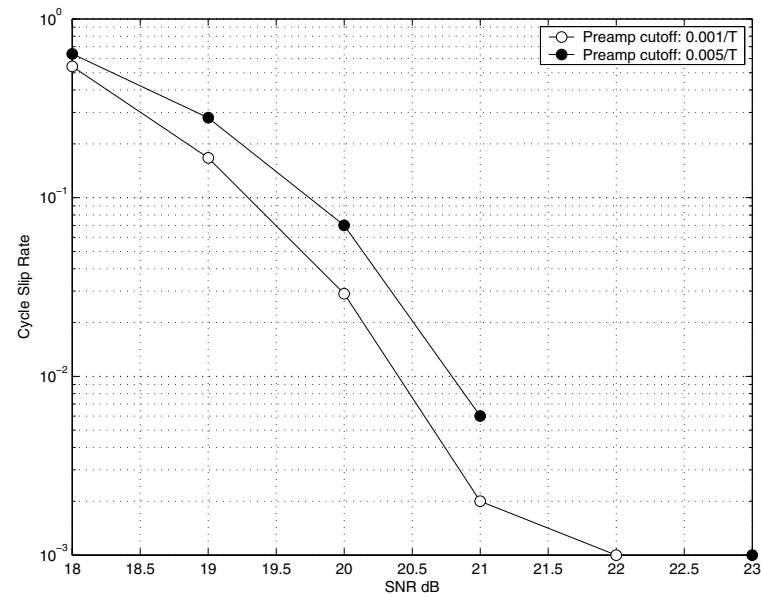

(b) Cycle slip rates.

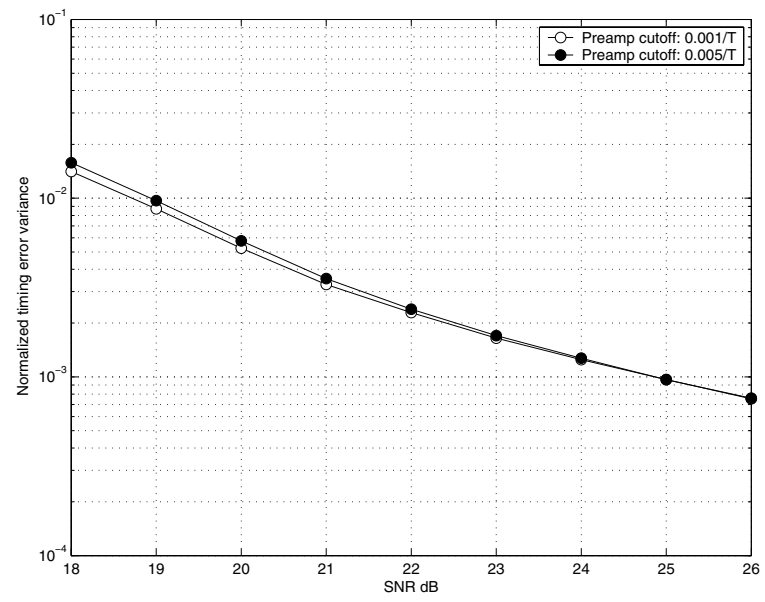

(c) Normalized timing error variances.

Figure 3. Comparison of timing recovery of two systems with different preamplifier cutoff frequencies and RLL encoded inputs.

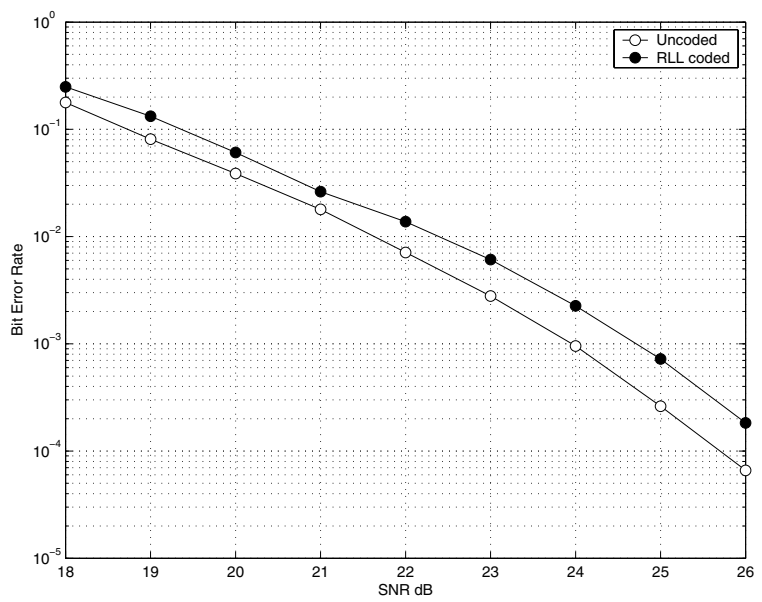

(a) Bit error rate.

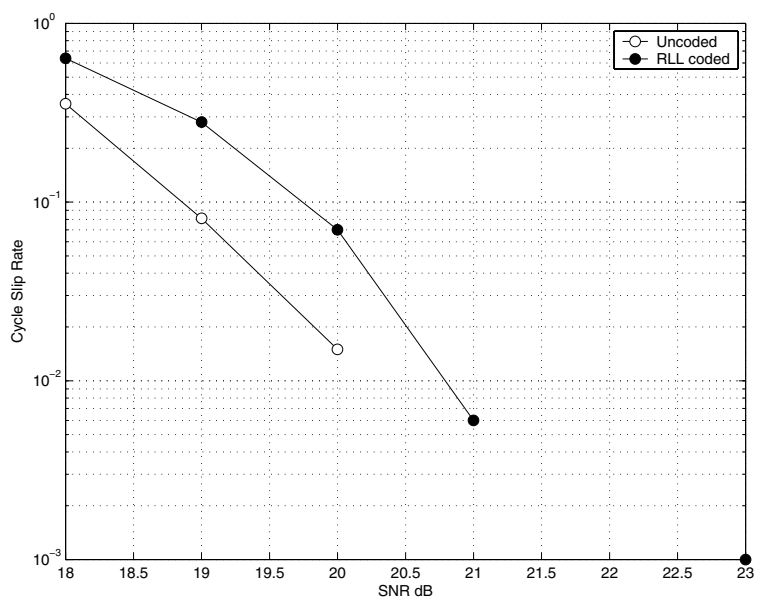

(b) Cycle slip rate.

Figure 4. Comparison of uncoded and RLL coded inputs with a preamplifier cutoff frequency of $0.005 / T$. 